\title{
Delayed plastic relaxation limit in SiGe islands grown by Ge diffusion from a local source
}

Cite as: J. Appl. Phys. 117, 104309 (2015); https://doi.org/10.1063/1.4914409

Submitted: 14 October 2014 . Accepted: 27 February 2015. Published Online: 13 March 2015

G. M. Vanacore, G. Nicotra, M. Zani, M. Bollani, E. Bonera, F. Montalenti, G. Capellini, G. Isella, J. Osmond, A. Picco, F. Boioli, and A. Tagliaferri

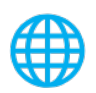

\section{ARTICLES YOU MAY BE INTERESTED IN}

Polycrystalline indium phosphide on silicon by indium assisted growth in hydride vapor phase epitaxy

Journal of Applied Physics 116, 033519 (2014); https://doi.org/10.1063/1.4890718

Electron trap level of hydrogen incorporated nitrogen vacancies in silicon nitride

Journal of Applied Physics 117, 104501 (2015); https://doi.org/10.1063/1.4914163

Boron and phosphorus diffusion in strained and relaxed Si and SiGe

Journal of Applied Physics 94, 3883 (2003); https://doi.org/10.1063/1.1602564

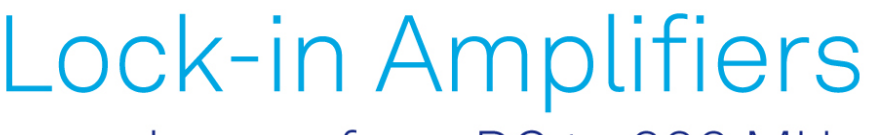

... and more, from DC to $600 \mathrm{MHz}$

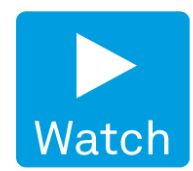




\title{
Delayed plastic relaxation limit in SiGe islands grown by Ge diffusion from a local source
}

\author{
G. M. Vanacore,,${ }^{1, a)}$ G. Nicotra, ${ }^{2}$ M. Zani, ${ }^{1}$ M. Bollani, ${ }^{3}$ E. Bonera,${ }^{4}$ F. Montalenti, ${ }^{4}$ \\ G. Capellini, ${ }^{5}$ G. Isella, ${ }^{6}$ J. Osmond, ${ }^{7}$ A. Picco, ${ }^{4}$ F. Boioli, ${ }^{4, b)}$ and A. Tagliaferri ${ }^{1, c}{ }^{4}$ \\ ${ }^{1}$ CNISM-Dipartimento di Fisica, Politecnico di Milano, Piazza Leonardo da Vinci 32, I-20133 Milano, Italy \\ ${ }^{2}$ IMM-CNR, Stradale Primosole 50, I-95121 Catania, Italy \\ ${ }^{3}$ CNR-IFN, LNESS, Via Anzani 42, I-22100 Como, Italy \\ ${ }^{4}$ Dipartimento di Scienza dei Materiali and L-NESS, Università Milano-Bicocca, via Cozzi 53, \\ I-20125 Milano, Italy \\ ${ }^{5}$ Department of Sciences at the Università Roma Tre, Via Vasca Navale 79, 00146 Roma, Italy \\ ${ }^{6}$ CNISM, LNESS, Dipartimento di Fisica, Politecnico di Milano (Polo di Como), Via Anzani 42, \\ I-22100 Como, Italy \\ ${ }^{7}$ ICFO-The Institute of Photonic Sciences, Av. Carl Friedrich Gauss, 3, E-08860 Castelldefels (Barcelona), Spain
}

(Received 14 October 2014; accepted 27 February 2015; published online 13 March 2015)

\begin{abstract}
The hetero-epitaxial strain relaxation in nano-scale systems plays a fundamental role in shaping their properties. Here, the elastic and plastic relaxation of self-assembled SiGe islands grown by surface-thermal-diffusion from a local Ge solid source on $\mathrm{Si}(100)$ are studied by atomic force and transmission electron microscopies, enabling the simultaneous investigation of the strain relaxation in different dynamical regimes. Islands grown by this technique remain dislocation-free and preserve a structural coherence with the substrate for a base width as large as $350 \mathrm{~nm}$. The results indicate that a delay of the plastic relaxation is promoted by an enhanced $\mathrm{Si}-\mathrm{Ge}$ intermixing, induced by the surface-thermal-diffusion, which takes place already in the SiGe overlayer before the formation of a critical nucleus. The local entropy of mixing dominates, leading the system toward a thermodynamic equilibrium, where non-dislocated, shallow islands with a low residual stress are energetically stable. These findings elucidate the role of the interface dynamics in modulating the lattice distortion at the nano-scale, and highlight the potential use of our growth strategy to create composition and strain-controlled nano-structures for new-generation devices. (C) 2015
\end{abstract} AIP Publishing LLC. [http://dx.doi.org/10.1063/1.4914409]

\section{INTRODUCTION}

The deposition of $\mathrm{Ge}$ on $\mathrm{Si}(100)$ surfaces follows the Stranski-Krastanov (SK) growth mode, ${ }^{1}$ where a twodimensional wetting layer (WL) is formed before the nucleation of three-dimensional islands. The main driving forces behind this process are nowadays well understood. ${ }^{2}$ Owing to its lower surface energy, Ge initially wets the $\mathrm{Si}$ surface forming a compressively strained WL. Ge grows in a layerby-layer mode up to a critical thickness of around four monolayers (ML), ${ }^{1,3}$ above which coherent stable islands are formed, lowering the total energy of the system with respect to the pure two-dimensional configuration. A transition of the island morphology with increasing Ge deposition has been experimentally observed, going from shallow small pyramids to steeper, larger domes, ${ }^{4}$ and to even steeper barn shaped islands. ${ }^{5}$ This evolution can be understood by considering that steeper islands can relax more effectively the elastic energy, at the expense of extra-surface energy with respect to the WL. The balance between these two terms yields to the following expression for the energy difference

\footnotetext{
${ }^{a)}$ Present address: Physical Biology Center for Ultrafast Science and Technology, Arthur Amos Noyes Laboratory of Chemical Physics, California Institute of Technology, Pasadena, California 91125, USA.

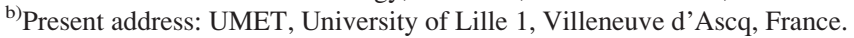

${ }^{c)}$ Author to whom correspondence should be addressed. Electronic mail: alberto.tagliaferri@polimi.it
}

between the island and a portion of flat wetting layer having equal volume, $V$ (Ref. 3)

$$
\Delta E=V\left(\rho_{i s}-\rho_{W L}\right)-V^{2 / 3}\left(c_{S}^{i s} \gamma_{i s}-c_{B}^{i s} \gamma_{W L}\right),
$$

where $i$ s refers to island, $S$ to surface, and $B$ to base. Here, $\rho_{i s}$ and $\rho_{W L}$ are the composition-dependent elastic energy densities in the island and in the WL; $\gamma_{i s}$ and $\gamma_{W L}$ the average surface energy densities of the island facets and of the WL; $c_{S}^{i s}$ and $c_{B}^{i s}$ the facets and base areas normalized to $V^{2 / 3}$, respectively. At large volumes, the surface energy term is negligible, so that steep islands are favored, while shallow morphologies are expected in the limit of small volume $V$. The experimental observation of a progressive transition towards islands with higher height-to-base aspect ratio (AR) with increasing volume, ${ }^{4,5}$ seems to confirm the qualitative prediction based on Eq. (1). However, this simple model is valid only under the assumption of a constant and uniform Ge concentration within the islands, and in the absence of plastic relaxation. A strain-driven $\mathrm{SiGe}$ intermixing ${ }^{6,7}$ and lateral and vertical composition gradients ${ }^{8}$ have been already clearly demonstrated, as well as plasticity onset beyond a critical volume. ${ }^{7,9-13}$ Both SiGe alloying and dislocation injection effectively lower the elastic energy of the islands by relieving the misfit strain. The tendency towards higher aspect ratios with increasing size predicted by Eq. (1) is interrupted by the nucleation of dislocations at the edge of an 
island. ${ }^{9}$ The lowering of elastic energy makes the island a sink for new material, and its shape flattens by an enhanced lateral expansion. This is experimentally evidenced by a discontinuity in the slope of the aspect ratio distribution as a function of the island base width, as reported in Ref. 7. The supply of Si to an already formed Ge island can also induce a "reversal" of the SK dynamics, leading to flatter islands. ${ }^{14}$ However, the kinetics of the growth process and the time scale over which the Si incorporation occurs, compared to the Ge supply, quantitatively influence the elemental composition and the structural coherence of the growing islands. Lattice distortions at the nano-scale play a fundamental role in shaping the optical and electronic properties of nanostructures. A direct control can be obtained only when the interplay between chemical composition, morphology and strain state, which represents one of the most intriguing aspects of $\mathrm{Ge} / \mathrm{Si}(100)$ SK growth, is fully understood.

In order to simultaneously investigate the strain relaxation over different dynamical regimes, we use a novel approach for the growth of self-assembled $\mathrm{SiGe}$ islands exploiting the thermally activated diffusion of Ge atoms from a local source. For this purpose, we have fabricated $\mathrm{Ge}$ stripes and annealed them in ultra-high vacuum (UHV) at different temperatures inducing Ge diffusion and islands self-assembly. As reported in previous works, ${ }^{15-17}$ this methodology has provided a unique approach for the investigation of the factors governing the growth process. From our previous results, ${ }^{17}$ we concluded that the island growth evolves in a diffusion-limited regime and it is mainly driven by the short-range interactions between the growing islands and the diffusing Ge atoms. Our observations also evidenced that, besides the Ge gathering from the surface, the Si incorporation from the substrate is similarly mediated by the kinetics of diffusion, thus imposing a strict constraint on the final volume and elastic strain state of the growing islands.

Here, we extend our analysis to the investigation of the strain relaxation dynamics, performed over a large range of island sizes at the growth temperatures of 600 and $700{ }^{\circ} \mathrm{C}$, and explore the limit of their plastic relaxation in comparison with deposition techniques. Using atomic force microscopy (AFM) and transmission electron microscopy (TEM) to investigate the morphology and the structure of single islands, we found that islands remain dislocation-free and preserve their structural coherence with the substrate for values of the base width in the $25-350 \mathrm{~nm}$ range. Our AFM and TEM results, combined with previous observations, ${ }^{17}$ reveal that the suppression of the plastic relaxation is strictly correlated with the tendency of the critical nuclei to grow by collecting simultaneously $\mathrm{Si}$ and Ge atoms in their surroundings. Moreover, our structural and morphological investigation conclusively indicates that, when kinetics of diffusion dominates, the intermixing is the dominant process for the strain relaxation, and leads the system toward its thermodynamic equilibrium. In this context, highly intermixed islands with a low residual stress and shallow morphologies are energetically favored to nucleate. This is distinct from the scenario obtained using deposition techniques (MBE and CVD), where only out-of-equilibrium, Gerich, small islands can remain coherent. These results show that the combination of local sources and a controlled nucleation can potentially be exploited to grow composition and strain-controlled SiGe structures to be used in nanoelectronic applications, ${ }^{18,19}$ such as nanofabricated stressors $^{20-23}$ and controlled-mobility channels.

\section{EXPERIMENT AND METHODS}

The details of the island growth by Ge surface diffusion from a local source have already been reported in previous works. ${ }^{15-17}$ Briefly, Ge stripes (width $\approx 5 \mu \mathrm{m}$, thickness $\approx 150 \mathrm{~nm}$ ) are obtained by a lithographic patterning of pure Ge thin films, deposed by Low Energy Plasma Enhanced Chemical Vapour Deposition (LEPECVD), ${ }^{24}$ and are annealed by Joule heating flowing a DC current through the $\mathrm{Si}(100)$ substrate. For the cases investigated here, the samples were annealed at $600^{\circ} \mathrm{C}$ for $15 \mathrm{~min}$ and at $700^{\circ} \mathrm{C}$ for $6 \mathrm{~min}$. Before the annealing, standard chemical and physical cleaning methods were used to obtain a clean and atomically flat surface. A PHI 660 Scanning Auger Electron Microscope (SAM) has been used for in-situ imaging and spatially resolved chemical analysis at the sample surface before and after thermal diffusion. Atomic Force Microscopy has been performed using a Veeco Innova microscope operating in tapping mode with ultra-sharp tips (nominal radius $\approx 2 \mathrm{~nm}$ ). Statistical analysis of the AFM data has been performed on more than one-thousand islands using freely available software tools. ${ }^{25}$ Transmission Electron Microscopy analysis has been obtained using a JEOL 2010 F TEM/STEM equipped with a $200 \mathrm{kV}$ Schottky field emission gun. Micro-Raman spectroscopy has been performed with a Jasco R800 spectrometer in backscattering geometry using $458 \mathrm{~nm}$ as excitation wavelength focused by a 0.9 numerical aperture objective.

\section{RESULTS AND DISCUSSION}

As we already reported in a previous publication, ${ }^{17}$ during the high-temperature annealing in UHV, Ge diffuses from the stripe on the Si surface forming a continuous overlayer (OL) (see schematics in Fig. 1(a)). SAM analysis ${ }^{15}$ shows that the Ge content of the over-layer is approximately constant within the nucleation region $(x<20 \mu \mathrm{m})$ and its average value decreases from $\sim 0.8$ at $600^{\circ} \mathrm{C}$ to $\sim 0.64$ at $700^{\circ} \mathrm{C}$. This temperature dependence reflects the thermally activated nature of the $\mathrm{Si}$ incorporation. When our results are compared to typical MBE data, where Ge concentration values of the wetting layer greater than 0.86 have been found even at $700{ }^{\circ} \mathrm{C},{ }^{26}$ it is evident that the thermal diffusion from a local source results in an enhanced intermixing within the OL, whose origin will be discussed in details later.

Spontaneous nucleation of self-assembled islands is observed to coexist with the continuous surface diffusion (see schematics in Fig. 1(a)). Fig. 1(b) shows representative AFM images of the islands nucleated at $600{ }^{\circ} \mathrm{C}$ and $700^{\circ} \mathrm{C}$. Besides their different size, a remarkable feature is the presence of a few nanometers deep trenches, made evident as light-grey shaded rings, around the islands grown at $600^{\circ} \mathrm{C}$, whereas no trenches are observed for islands grown at $700{ }^{\circ} \mathrm{C}$. This is opposite to the behavior observed for islands grown by "standard" deposition techniques, where deeper trenches are found around larger islands, ${ }^{27}$ and it is strictly 

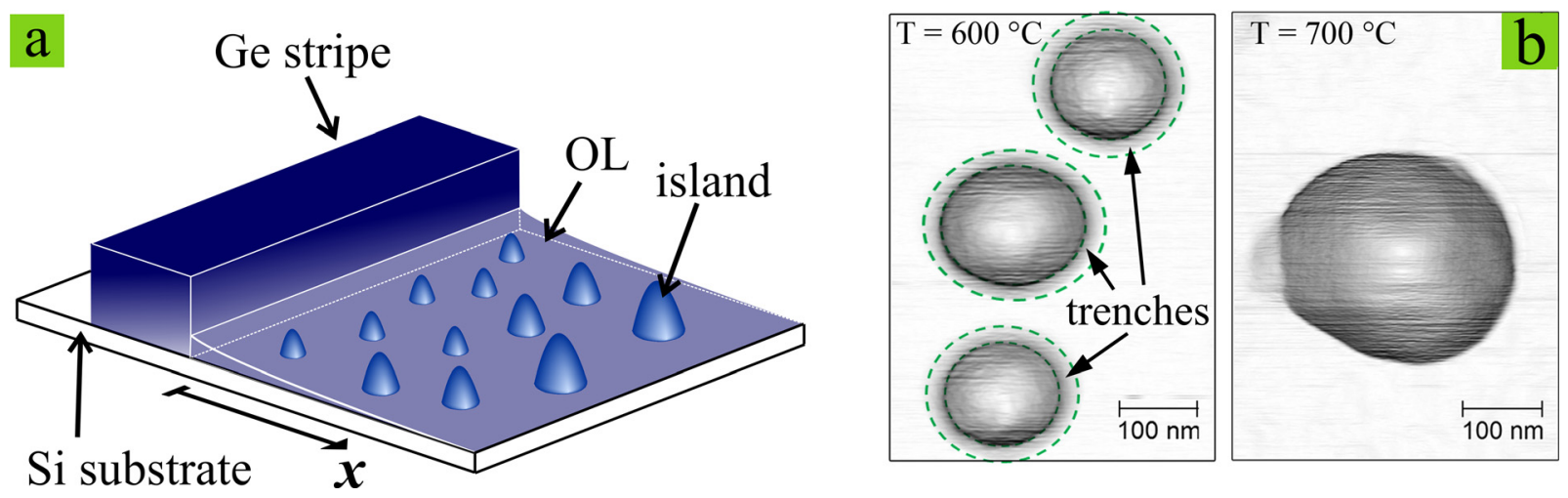

FIG. 1. Ge diffusion and island morphology. (a) Schematic representation of the OL formation and island growth by Ge diffusion from a local source after high-temperature annealing. (b) AFM images in gradient mode of the investigated islands grown at $600{ }^{\circ} \mathrm{C}$ (left), and at $700^{\circ} \mathrm{C}$ (right). The dashed lines define the border of the trenches visible around the islands. The islands shown are representatives of the whole island population.

related to the interplay between the Ge and Si supply, as discussed below.

As described by De Seta et al., ${ }^{7}$ an indication on the structural state of the islands can be provided by the distribution of their aspect ratio as a function of the base width, $b$, which shows a discontinuity in slope when the strain relaxation by the nucleation of dislocation occurs (see Ref. 7). A similar graph is shown in Fig. 2 for islands grown by surface-thermal-diffusion at $600^{\circ} \mathrm{C}$ and $700^{\circ} \mathrm{C}$. The monotonic increase of the aspect ratio with $b$ is consistent with a continuous transition from shallow to steeper morphologies. This allows for a partial elastic strain relief, as qualitatively described by Eq. (1). The lack of any slope change in the aspect ratio distribution indicates that, in average, islands are preserving their structural coherence with the substrate (for a base width as large as $350 \mathrm{~nm}$ at $700^{\circ} \mathrm{C}$ ), and the plastic relaxation channel, mediated by injection of misfit dislocation, is quenched.

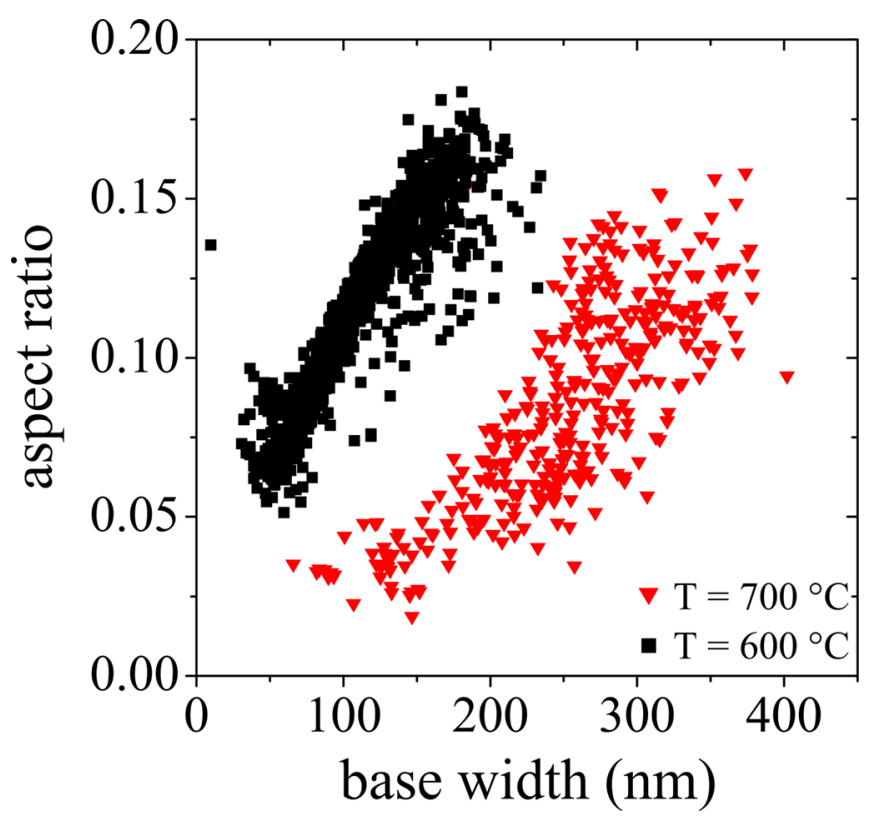

FIG. 2. Aspect ratio distribution. Aspect ratio (height/base-width) distribution as a function of the base width, defined as the square root of the projected area, for islands grown at $600^{\circ} \mathrm{C}$ (black squares) and $700{ }^{\circ} \mathrm{C}$ (red triangles).
To confirm our observations, we performed a direct non-averaged, nano-scale structural analysis of the islands by TEM. The plastic relaxation, following the injection of dislocations, can be experimentally evidenced by the presence of moiré and cross-hatched patterns (see for instance Ref. 28), as shown in Figs. 3(a)-3(d) where we display TEM images acquired on the plastically relaxed Ge stripe. The comparison between these images and those of representative islands nucleated in the diffusion region (Figs. 3(e)-3(h)) allows us to draw stringent conclusions on their plastic relaxation. In the sample annealed at $700^{\circ} \mathrm{C}$, the investigated islands have base width values ranging between $\sim 115$ and $\sim 275 \mathrm{~nm}$, which represent a characteristic portion of the island size distribution, and have nucleated at a stripe distance between $\sim 2$ and $\sim 7 \mu \mathrm{m}$.

Figs. 3(e) and 3(g) show plan-view bright-field TEM images of single islands grown at $700^{\circ} \mathrm{C}$, acquired in [001] axis beam condition (to be compared with Figs. 3(a) and $3(b))$ and in a relatively thick region, such that we can exclude a relaxation of the lamella, far from the amorphized portion of the substrate, as confirmed by the presence of diffraction contrast surrounding the islands. The (almost) circular diffraction fringes visible within the islands indicate a lateral outward bending of the lattice planes, consistent with an elastic strain relaxation. The absence of moire patterns excludes a plastic relaxation, in agreement with the indication derived from the aspect ratio distribution, and indicates that the islands still preserve a structural coherence with the $\mathrm{Si}$ substrate. This also is clearly visible in the TEM image taken in [022] diffraction condition (Fig. 3(f), to be compared with Figs. 3(c) and 3(d)), showing a uniform contrast within the island, whereas the presence of dislocations would generate characteristic dark fringes. It is worth noting that the black regions visible on the lower side of the island in Fig. 3(f) are due to lattice plane families in Bragg condition with the electron beam, and are observed to move from one side to the other by changing the diffraction angle.

The absence of crystalline defects is also verified by low angle annular dark field (LAADF) scanning-TEM imaging (Fig. 3(h)), where the contrast is mainly determined by the atomic number of the elements. In this imaging mode, a dislocation would appear as an intense white line due to the 

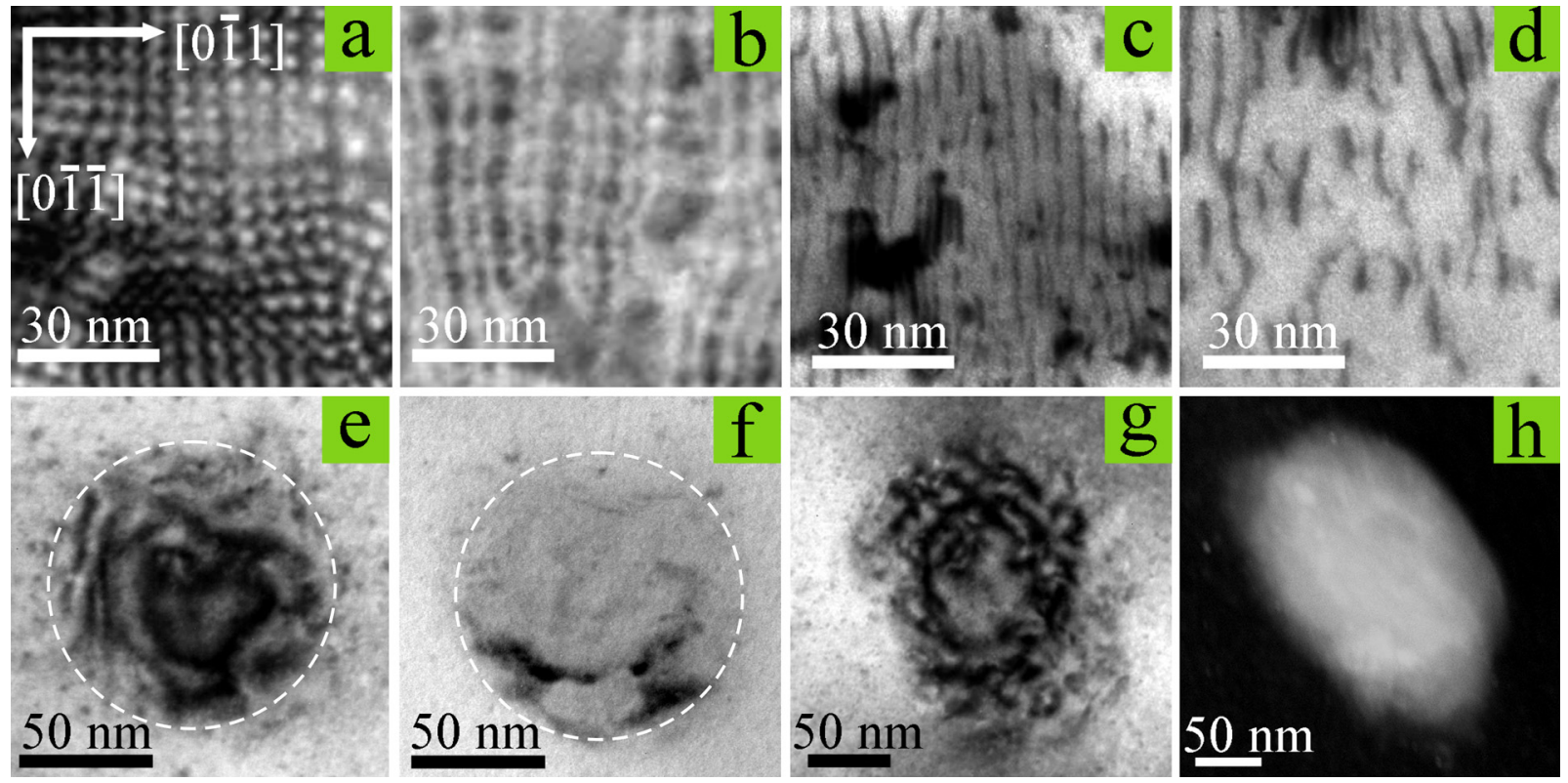

FIG. 3. TEM structural analysis for islands grown at $700^{\circ} \mathrm{C}$. (a)-(d) TEM images acquired on the relaxed Ge stripe. (e) and (f) TEM images of SiGe islands grown at $700^{\circ} \mathrm{C}$. All images are aligned according to the directions shown in (a). (a) and (b) Plan-view, bright-field images of the Ge stripe under [100] diffraction condition. The panel (a) shows the moiré patterns due to the misfit at the interface between the relaxed stripe and the Si bulk, whereas the panel (b) represents the cross-hatched pattern due to the $90^{\circ}$ edge dislocations propagating along the interface. (c) and (d) Weak-beam, bright-field images of the Ge stripe in [022] diffraction condition. The lines parallel to the [011] direction shown in (c) are due to the $90^{\circ}$ dislocations. The short and irregulars segments in (d) come from the threading arms of the $60^{\circ}$ dislocations. (e)-(g) Plan-view, bright-field images of SiGe islands under [100] zone-axis (same experimental conditions as panels (a) and (b)). (f) Weak-beam, bright-field image under [022] zone-axis (same experimental conditions as panels (c) and (d)) of the island shown in (e). The white dashed lines in (e) and (f) delimit the island shape. (h) LAADF scanning-TEM image of a large dislocation-free island.

random displacement of the atoms around the defect, causing extra scattering in the low angle region. ${ }^{29}$ Fig. 3(h) shows the STEM image of a large island (base width $\approx 275 \mathrm{~nm}$ ), which appears as a nearly uniform lighter area, and the absence of brighter linear features aligned along the $\langle 011\rangle$ directions excludes the presence of dislocations or stacking faults.

The picture described so far for islands grown at $700^{\circ} \mathrm{C}$ is also valid for the case of annealing at lower temperatures. Fig. 4 shows plan-view bright-field TEM images under [02 $\overline{2}]$ axis beam condition of islands grown at $600^{\circ} \mathrm{C}$. The analysis has been performed in a $2.3 \mu \mathrm{m}$-wide region beside the stripe (much larger than the denuded zone of $\sim 150 \mathrm{~nm}$ ), where a representative ensemble of islands with dimensions ranging between $\sim 40$ and $\sim 170 \mathrm{~nm}$ can be found. On one hand, islands with base width values smaller than $\sim 150 \mathrm{~nm}$, which represent the majority of the nucleated ones, appear to be dislocation-free as seen by the uniform contrast in the TEM images (see Fig. 4(a)). On the other hand, some larger islands (base width larger than $\sim 150 \mathrm{~nm}$ ) exhibit multiple fringes (see for instance Fig. 4(b)), whose presence could be related to the occurrence of crystal defects. Once again, the indication derived by the aspect ratio distribution about the structural coherence of the islands is mainly confirmed by TEM measurements, although the presence of defects in large islands do not allow us to exclude that some of them could already be plastically relaxed or close to the critical value for the insertion of a dislocation.

To elucidate the origin of the observed behavior, the energetics of the growth process has to be discussed. The driving force for the injection of misfit dislocations is the decrease of the elastic energy stored into the island. Si-Ge intermixing leads to a similar effect, since it lowers the effective misfit with the substrate, and minimizes the energy

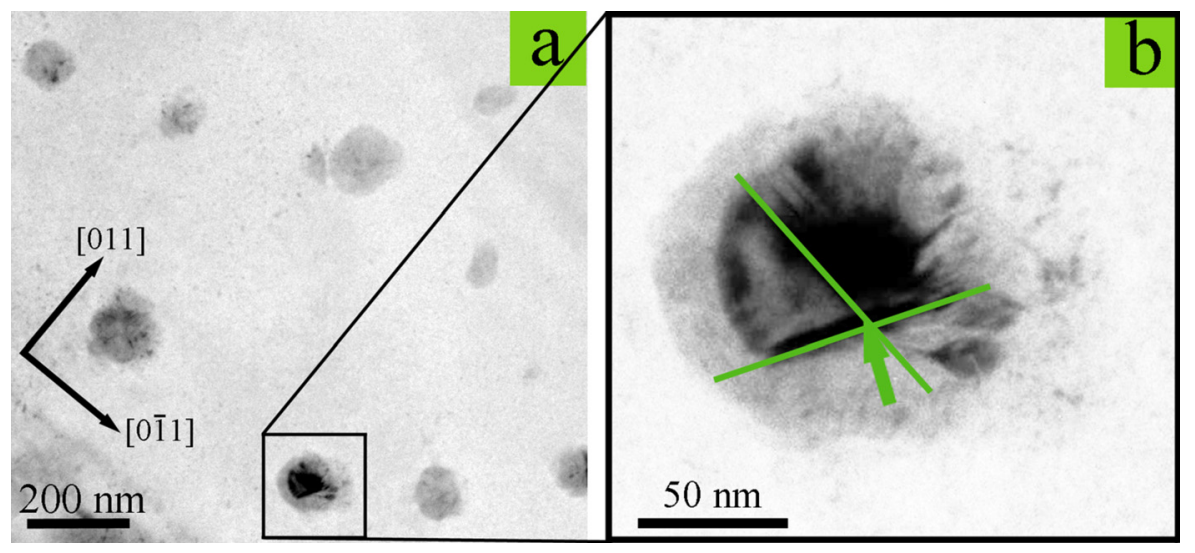

FIG. 4. TEM structural analysis for islands grown at $600^{\circ} \mathrm{C}$. (a) and (b) Representative plan-view, bright-field TEM images under [02 $\overline{2}]$ zone-axis of SiGe islands grown at $600^{\circ} \mathrm{C}$. The majority of the islands appears to be dislocation-free, since a uniform contrast is observed in the images, although some large islands exhibits crystal defects, such as the twins indicated by the green arrow observed for the large island magnified in the panel (b). 
component related to the entropy of mixing ${ }^{30,31}$ within the total free energy balance. Thus, intermixing and dislocation injection influence each other: islands grown at higher temperatures, being richer in $\mathrm{Si}$, will display a lower tendency towards plastic relaxation, leading to larger critical size for injection of dislocations. ${ }^{7,32,33}$ The interplay among strain relaxation, $\mathrm{Si}-\mathrm{Ge}$ intermixing, and size evolution has to be then carefully addressed.

As reported in a previous publication, ${ }^{17}$ the island size is strictly correlated to the distance from the stripe, $x$. Apart from the region very close to the stripe, where the growth is determined by the competition between the preferential nucleation at the stripe edge and the high Ge flux from the source, the island size progressively increases moving away from the stripe. This trend is determined by the coupling between nucleation dynamics, intermixing, and Ge coverage, which directly influence the strain relaxation of the islands.

The composition of single islands as a function of the distance from the stripe has been measured using microRaman spectroscopy. While this analysis has also been employed in a previous work ${ }^{17}$ to investigate the nucleation regime, here is used instead to discuss the strain relaxation dynamics. At $700^{\circ} \mathrm{C}$, the measured Ge content within the islands changes from 0.45 to about 0.25 moving away from the stripe (for $x>2 \mu \mathrm{m}$ ), with an experimental uncertainty of $\sim 0.03$. These values are obtained using Raman shift coefficients determined on biaxially strained thin films, ${ }^{34}$ which are uniform in strain and composition. Although this is not the case for the islands, which are inhomogeneous in both composition $^{8}$ and strain, ${ }^{33}$ this method has been proved valid

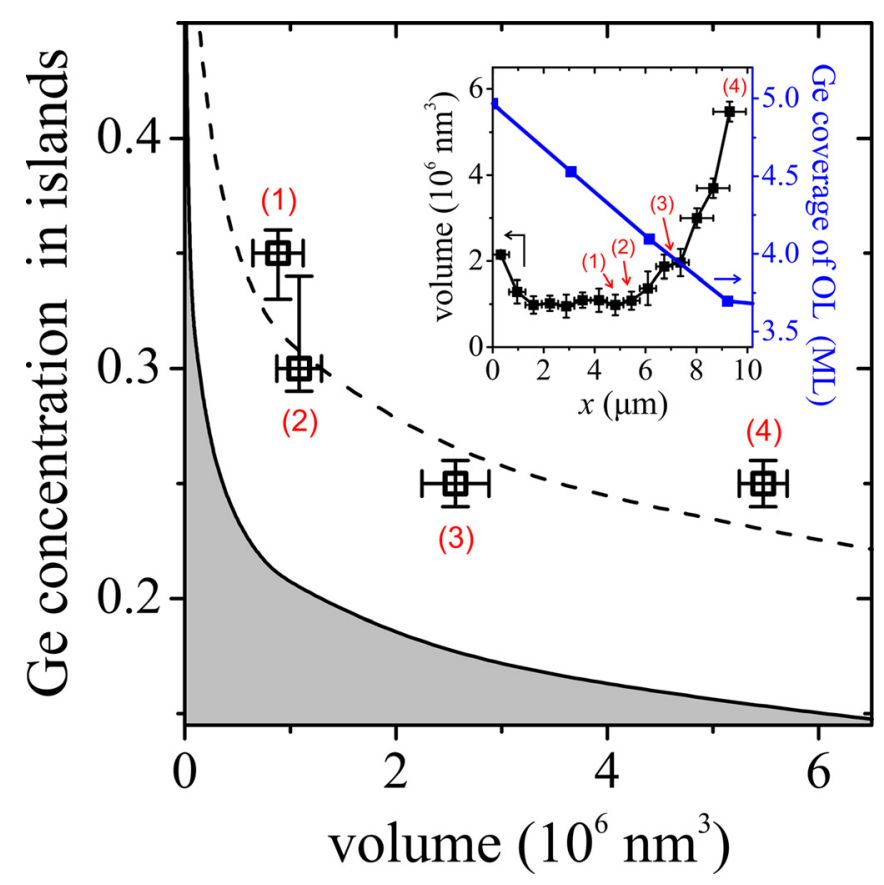

FIG. 5. Evolution of island size and composition. Observed correlation between island volume and island composition, together with the calculated composition-dependent critical volume for a SiGe dome (black solid line). The light-grey area defines the region of thermodynamic instability and the dashed black line is a guide to the eye. Inset: island volume (black symbols) and Ge coverage of the diffused over-layer (blue symbols) as a function of the distance from the stripe (data from Ref. 17). The size and position of the islands in the main panel are indicated by the arrows. for the determination of the average values of strain and composition, as discussed for instance in Refs. 35 and 36. The Ge concentration within the islands exhibits a clear variation as a function of the distance from the stripe, which is well above the experimental uncertainty.

In Fig. 5, the observed correlation between island size and island composition is shown for the case of annealing at $700^{\circ} \mathrm{C}$, together with the calculated composition-dependent critical volume for a SiGe dome, whereas in the inset, we represent the dependence of the island volume and the $\mathrm{Ge}$ coverage of the diffused over-layer on the distance from the stripe. From these trends, a higher Si content is evident for larger islands nucleated farther away from the stripe, where the Ge coverage is lower. As also discussed in Ref. 17, this indicates that close to the stripe edge, the high Ge flux kinetically limits the $\mathrm{Si}$ incorporation within the growing islands and only small islands are energetically allowed to nucleate. Away from the stripe, the decreased Ge flux promotes a lower density of supercritical nuclei, leaving larger areas from which adatoms can be collected. This favors the incorporation of $\mathrm{Si}$ atoms coming from the substrate, which acts as a local solid source, and leads to an increase of the average island size. ${ }^{7,17}$ The local kinetics of diffusion is thus dominating the growth process.

Since the Ge supply from the stripe occurs on a time scale longer than the Si incorporation from the substrate, the presence of a remote source intrinsically favors a larger tendency for a Si-Ge mixing during the island growth. In this scenario, our AFM and TEM results (Figs. 2-4), combined with previous observations (Fig. 5) conclusively indicate that the intermixing becomes the dominant process for the strain relaxation, leading to the quenching of the plastic channel and thus preventing the injection of misfit dislocations. At $700^{\circ} \mathrm{C}$, we found that the large coherent islands imaged by TEM $(b \approx 275 \mathrm{~nm})$ and AFM $(b \approx 350 \mathrm{~nm})$ have an average Ge concentration, $c_{\mathrm{Ge}}$, of $\sim 0.25-0.3$, as obtained by microRaman. These values of $b$ and $c_{\mathrm{Ge}}$ have to be compared with the critical base width, $b_{\text {crit }}$, and the Ge content at the plastic relaxation onset for islands grown at $700^{\circ} \mathrm{C}$ by "standard" deposition techniques. For $\mathrm{MBE},{ }^{32}$ we have $b_{\text {crit }} \approx 80 \mathrm{~nm}$ and $c_{\mathrm{Ge}} \approx 0.4$, whereas for $\mathrm{CVD}^{7}{ }^{7} b_{\text {crit }} \approx 200 \mathrm{~nm}$ and $c_{\mathrm{Ge}} \approx 0.5$. Therefore, we can quantitatively assess that in our case the plastic relaxation mechanism is delayed because of the increased intermixing. It is worth mentioning that coherent islands as large as $\sim 350-400 \mathrm{~nm}$ have also been reported in the case of $\mathrm{CVD}^{7}$ and $\mathrm{MBE}^{37}$ growth. However, this condition has been reached only at very high growth temperatures, $\mathrm{T} \sim 850-900^{\circ} \mathrm{C}$, where side effects such as bulk inter-diffusion and Ge desorption become relevant, which can drastically perturb the optical and electronic properties of the nanostructures.

Our observations also reveal that when a remote $\mathrm{Ge}$ source is used, the microscopic mechanism behind the $\mathrm{Si}-\mathrm{Ge}$ intermixing changes as compared with deposition techniques. In MBE, a Ge-rich wetting layer $\left(c_{G e}^{W L}>0.86\right)$ forms even at high temperatures $\left(\mathrm{T} \approx 700^{\circ} \mathrm{C}\right) .{ }^{26}$ When the $\mathrm{WL}$ thickness exceeds its critical value, faceted Ge-rich islands nucleate and grow at the expense of smaller ones via a coarsening process. ${ }^{38}$ At this point, trenches form around the 
islands, digging deeply into the substrate. The ejected $\mathrm{Si}$ mixes with the Ge islands leading to the formation of alloyed nanocrystals. ${ }^{31}$ Within this scenario, Brehm et al. ${ }^{37}$ have observed that an evolution toward larger islands with higher aspect ratio can be obtained by increasing the growth temperature.

In the case here discussed, the scenario is quite different. At $700^{\circ} \mathrm{C}$, the combination of several elements, such as: (i) the presence of a highly intermixed over-layer $\left(c_{G e}^{O L} \approx 0.64\right)$, (ii) the dominant contribution of the local kinetics of diffusion during the growth (see Fig. 5), and (iii) the lack of lateral trenches (see the right panel in Fig. 1(b)), indicates that the critical nuclei grow by gathering simultaneously Si and $\mathrm{Ge}$ in their surroundings. In this scenario, the intermixing takes place from the very beginning, as soon as the continuous over-layer is created and thus before a critical nucleus is formed. The ensemble of islands is now closer to its thermodynamic limit, where entropy of mixing dominates, and highly intermixed islands with a low residual stress would be energetically favored to nucleate. This is experimentally confirmed by micro-Raman spectroscopy, from which we obtained for the large coherent islands imaged by TEM and AFM a Ge content as low as $25 \%$ and an average strain varying between $0.1 \times 10^{-2}$ and $-0.2 \times 10^{-2}$, with an experimental uncertainty of $0.2 \times 10^{-2}$. The high-Si content and the low-strain are able to lower the atomic mobility around the islands, and because the $\mathrm{Si}$ incorporation now involves their entire volume, trenches are no longer needed to mediate the intermixing.

Within this picture, at high annealing temperatures, the low residual stress within the islands leads to a negligible elastic term in the energy balance of Eq. (1), and thus, the ensemble of islands tends to assume shallow morphologies in order to minimize the surface energy, similarly to the "reversed" SK dynamics observed during Si capping. ${ }^{14}$ This is experimentally confirmed by the observed lower aspect ratio for islands grown at $700{ }^{\circ} \mathrm{C}$ compared to those formed at $600{ }^{\circ} \mathrm{C}$ (see Fig. 2), contrary to MBE-islands where an increasing aspect ratio is observed for higher growth temperatures. ${ }^{37}$

When the growth by thermal-diffusion is performed at lower annealing temperatures, a MBE-like intermixing process is partially recovered. At $600^{\circ} \mathrm{C}$, a lower critical base width is observed $(\sim 150-175 \mathrm{~nm}$ from TEM and AFM), which is consistent with a reduced tendency of mixing between $\mathrm{Si}$ and $\mathrm{Ge}^{39,40}$ The observed presence of structural defects in islands smaller than those nucleated at $700^{\circ} \mathrm{C}$ (see Fig. 4) indicates a less efficient elastic strain relief, as caused by a decreased $\mathrm{Si}$ incorporation, which becomes mainly mediated by shallow trenches formed around the islands (see the left panel in Fig. 1(b)).

\section{CONCLUSIONS}

We have experimentally studied the interplay between plastic relaxation and Si-Ge intermixing in self-assembled $\mathrm{SiGe}$ islands grown by surface-thermal-diffusion from a local Ge solid source on a $\mathrm{Si}(100)$ surface. The aspect ratio distribution as a function of the base width gives a statistical indication that the nucleated islands preserve their structural coherence with the substrate for base width values as large as $350 \mathrm{~nm}$, as derived by the AFM analysis. The absence of extended defects has been confirmed by nano-scale structural analysis performed with TEM on single islands, where the absence of moiré patterns and electron de-channeling effects excludes a plastic relaxation by injection of misfit dislocations. We found that the suppression of the plastic relaxation occurs when the critical nuclei grow by gathering simultaneously $\mathrm{Si}$ and $\mathrm{Ge}$ in their surroundings. This process is favored by the local kinetics of diffusion and entropy of mixing, generating non-dislocated, shallow islands with a low residual stress. This is distinct from the scenario obtained using "standard" deposition techniques (MBE and CVD), where only out-of-equilibrium, Ge-rich, small islands can remain coherent. These results indicate that the use of a local Ge source can provide a wide range of tunability for composition and strain-controlled SiGe structures, to be used in nano-electronic applications.

\section{ACKNOWLEDGMENTS}

We acknowledge the Cariplo Foundation within the grant DefCon4 2011-0331 and the EIDOS 2011-0382 Project for partial financial support. The TEM work was performed at Beyond-Nano CNR-IMM, which is supported by the Italian Ministry of Education and Research (MIUR) under project Beyond-Nano (PON a3_00363).

${ }^{1}$ Y. W. Mo, D. E. Savage, B. S. Swartzentruber, and M. G. Lagally, Phys. Rev. Lett. 65, 1020 (1990).

${ }^{2}$ J.-N. Aqua, I. Berbezier, L. Favre, T. Frisch, and A. Ronda, Phys. Rep. 522, 59 (2013).

${ }^{3}$ M. Brehm, F. Montalenti, M. Grydlik, G. Vastola, H. Lichtenberger, N. Hrauda, M. J. Beck, T. Fromherz, F. Schäffler, L. Miglio, and G. Bauer, Phys. Rev. B 80, 205321 (2009).

${ }^{4}$ G. Medeiros-Ribeiro, A. M. Bratkovski, T. I. Kamins, D. A. A. Ohlberg, and R. S. Williams, Science 279, 353 (1998).

${ }^{5}$ M. Stoffel, A. Rastelli, J. Tersoff, T. Merdzhanova, and O. Schmidt, Phys. Rev. B 74, 155326 (2006).

${ }^{6}$ S. A. Chaparro, J. Drucker, Y. Zhang, D. Chandrasekhar, M. R. McCartney, and D. J. Smith, Phys. Rev. Lett. 83, 1199 (1999).

${ }^{7}$ M. De Seta, G. Capellini, F. Evangelisti, and C. Spinella, J. Appl. Phys. 92, 614 (2002).

${ }^{8}$ A. Rastelli, M. Stoffel, A. Malachias, T. Merdzhanova, G. Katsaros, K. Kern, T. Metzger, and O. G. Schmidt, Nano Lett. 8, 1404 (2008).

${ }^{9}$ F. K. LeGoues, M. C. Reuter, J. Tersoff, M. Hammar, and R. M. Tromp, Phys. Rev. Lett. 73, 300 (1994).

${ }^{10}$ T. Merdzhanova, S. Kiravittaya, A. Rastelli, M. Stoffel, U. Denker, and O. G. Schmidt, Phys. Rev. Lett. 96, 226103 (2006).

${ }^{11}$ M.-I. Richard, T. U. Schülli, and G. Renaud, Appl. Phys. Lett. 99, 161906 (2011).

${ }^{12}$ M.-I. Richard, A. Malachias, J.-L. Rouvière, T.-S. Yoon, E. Holmström, Y.-H. Xie, V. Favre-Nicolin, V. Holý, K. Nordlund, G. Renaud, and T.-H. Metzger, Phys. Rev. B 84, 075314 (2011).

${ }^{13}$ H. Ye, Z. Yu, P. Lu, Y. Liu, and L. Han, J. Appl. Phys. 114, 093504 (2013).

${ }^{14}$ G. Capellini, M. De Seta, L. Di Gaspare et al., J. Appl. Phys. 98, 124901 (2005).

${ }^{15}$ G. M. Vanacore, M. Zani, G. Isella, J. Osmond, M. Bollani, and A. Tagliaferri, Phys. Rev. B 82, 125456 (2010).

${ }^{16}$ G. M. Vanacore, M. Zani, M. Bollani, D. Colombo, G. Isella, J. Osmond, R. Sordan, and A. Tagliaferri, Nanoscale Res. Lett. 5, 1921 (2010).

${ }^{17}$ G. M. Vanacore, M. Zani, M. Bollani, E. Bonera, G. Nicotra, J. Osmond, G. Capellini, G. Isella, and A. Tagliaferri, Nanotechnology 25, 135606 (2014). 
${ }^{18}$ G. Katsaros, P. Spathis, M. Stoffel, F. Fournel, M. Mongillo, V. Bouchiat, F. Lefloch, A. Rastelli, O. G. Schmidt, and S. De Franceschi, Nat. Nanotechnol. 5, 458 (2010).

${ }^{19}$ F. A. Zwanenburg, A. S. Dzurak, A. Morello, M. Y. Simmons, L. C. L. Hollenberg, G. Klimeck, S. Rogge, S. N. Coppersmith, and M. A. Eriksson, Rev. Mod. Phys. 85, 961 (2013).

${ }^{20}$ D. Chrastina, G. M. Vanacore, M. Bollani, P. Boye, S. Schöder, M. Burghammer, R. Sordan, G. Isella, M. Zani, and A. Tagliaferri, Nanotechnology 23, 155702 (2012).

${ }^{21}$ G. M. Vanacore, M. Chaigneau, N. Barrett, M. Bollani, F. Boioli, M. Salvalaglio, F. Montalenti, N. Manini, L. Caramella, P. Biagioni, D. Chrastina, G. Isella, O. Renault, M. Zani, R. Sordan, G. Onida, R. Ossikovski, H.-J. Drouhin, and A. Tagliaferri, Phys. Rev. B 88, 115309 (2013).

${ }^{22}$ G. M. Vanacore, Nuovo Cimento C 35, 49-58 (2012).

${ }^{23}$ E. Bonera, M. Bollani, D. Chrastina, F. Pezzoli, A. Picco, O. G. Schmidt, and D. Terziotti, J. Appl. Phys. 113, 164308 (2013).

${ }^{24}$ G. Isella, D. Chrastina, B. Rössner, T. Hackbarth, H.-J. Herzog, U. König, and H. von Känel, Solid-State Electron. 48, 1317 (2004).

${ }^{25}$ See www.gwyddion.net for GWYDDION.

${ }^{26}$ M. Brehm, M. Grydlik, H. Lichtenberger, T. Fromherz, N. Hrauda, W. Jantsch, F. Schäffler, and G. Bauer, Appl. Phys. Lett. 93, 121901 (2008).

${ }^{27}$ S. A. Chaparro, Y. Zhang, and J. Drucker, Appl. Phys. Lett. 76, 3534 (2000).
${ }^{28}$ G. Capellini, M. De Seta, Y. Busby, M. Pea, F. Evangelisti, G. Nicotra, C. Spinella, M. Nardone, and C. Ferrari, J. Appl. Phys. 107, 063504 (2010).

${ }^{29}$ Y. Zhiheng, D. A. Muller, and J. Silcox, J. Appl. Phys. 95, 3362 (2004).

${ }^{30}$ G. Medeiros-Ribeiro and R. S. Williams, Nano Lett. 7, 223 (2007).

${ }^{31}$ Y. Tu and J. Tersoff, Phys. Rev. Lett. 98, 096103 (2007).

${ }^{32}$ A. Marzegalli, V. A. Zinovyev, F. Montalenti, A. Rastelli, M. Stoffel, T. Merdzhanova, O. G. Schmidt, and Leo Miglio, Phys. Rev. Lett. 99, 235505 (2007).

${ }^{33}$ R. Gatti, A. Marzegalli, V. A. Zinovyev, F. Montalenti, and L. Miglio, Phys. Rev. B 78, 184104 (2008).

${ }^{34}$ F. Pezzoli, E. Bonera, E. Grilli, M. Guzzi, S. Sanguinetti, D. Chrastina, G. Isella, H. von Känel, E. Wintersberger, J. Stangl, and G. Bauer, J. Appl. Phys. 103, 093521 (2008).

${ }^{35}$ A. Picco, E. Bonera, F. Pezzoli, E. Grilli, O. G. Schmidt, F. Isa, S. Cecchi, and M. Guzzi, Nanoscale Res. Lett. 7, 633 (2012).

${ }^{36}$ E. Bonera, F. Pezzoli, A. Picco, G. Vastola, M. Stoffel, E. Grilli, M. Guzzi, A. Rastelli, O. G. Schmidt, and L. Miglio, Phys. Rev. B 79, 075321 (2009).

${ }^{37}$ M. Brehm, H. Lichtenberger, T. Fromherz, and G. Springholz, Nanoscale Res. Lett. 6, 70 (2011).

${ }^{38}$ F. Ross, R. Tromp, and M. Reuter, Science 286, 1931 (1999).

${ }^{39}$ G. Capellini, M. De Seta, and F. Evangelisti, Appl. Phys. Lett. 78, 303 (2001).

${ }^{40}$ M. Floyd, Y. Zhang, K. P. Driver, J. Drucker, P. A. Crozier, and D. J. Smith, Appl. Phys. Lett. 82, 1473 (2003). 